Published as:

Nardi T., Leterrier Y., Karimi A., Månson J.-A.E., A Novel Synthetic Strategy for Bioinspired Functionally Graded Nanocomposites Employing Magnetic Field Gradients, RSC Adv., 4, 72467255 (2014). DOI 10.1039/C3RA46731G. @ 2014. This manuscript version is made available under the CC-BY-NC-ND 4.0 license http://creativecommons.org/licenses/by-nc-nd/4.0/

\title{
A NOVEL SYNTHETIC STRATEGY FOR BIOINSPIRED FUNCTIONALLY GRADED NANOCOMPOSITES EMPLOYING MAGNETIC FIELD GRADIENTS
}

\author{
Tommaso Nardi ${ }^{a}$, Yves Leterrier ${ }^{a *}$, Ayat Karimi ${ }^{\mathrm{b}}$ and Jan-Anders E. Månson ${ }^{\mathrm{a}}$ \\ ${ }^{a}$ Laboratoire de Technologie des Composites et Polymères (LTC) \\ École Polytechnique Fédérale de Lausanne (EPFL) \\ CH-1015, Lausanne, Switzerland \\ ${ }^{b}$ Laboratoire de Physique de la Matière Complexe (LPMC) \\ École Polytechnique Fédérale de Lausanne (EPFL) \\ CH-1015, Lausanne, Switzerland
}

(*) corresponding author

E-mail: yves.leterrier@epfl.ch; Fax:+41 2169 35880; Tel: +41 216934848

\begin{abstract}
In order to mimic the complex architecture of many bio-materials and synthesize composites characterized by continuously graded composition and mechanical properties, an innovative synthetic strategy making use of magnetic field gradients and based on the motion of superparamagnetic $\mathrm{Fe}_{3} \mathrm{O}_{4} @ \mathrm{SiO}_{2}$ core-shell nanoparticles is adopted. It is demonstrated that by lowering the viscosity of the system through particle functionalization, and increasing the magnetic force acting on the nanoparticles upon optimization of a simple set-up composed of two permanent magnets in repulsion configuration, the magnephoretic process can be considerably accelerated. Thus, owing to the magnetic responsiveness of the $\mathrm{Fe}_{3} \mathrm{O}_{4}$ core and the remarkable mechanical properties of the $\mathrm{SiO}_{2}$ shell, approximately $150 \mu \mathrm{m}$ thick polymeric films with continuous gradients in composition and characterized by considerable increments in elastic modulus (up to $\approx 70 \%$ ) and hardness (up to $\approx 150 \%$ ) when going from particle-depleted to particle-enriched regions can be synthesized, even in times as short as 1 hour. The present methods are highly promising for a more efficient magnetic force-based synthesis of inhomogeneous soft materials whose composition is required to be locally tuned to meet the specific mechanical demands arising from non-uniform external loads.
\end{abstract}

\section{Introduction}

Many bio-composites such as bamboo, ${ }^{1}$ tissue interfaces, ${ }^{2,3}$ teeth $^{4}$ and the majority of exoskeletal structures ${ }^{5,6}$ exemplify how tailoring and optimization of their mechanical performance rely strongly on the creation of specific hierarchical architectures and on the accurate spatial distribution of readily available reinforcing elements. It has been shown, for example, how mollusk shells improve their resistance to contact damage 
and layer delamination and provide a barrier to crack propagation through a smart spatial design of elastic moduli, which undergo approximately $40 \%$ gradual changes between intercrystalline soft organic layers and stiff ceramic crystallites. ${ }^{7}$ Over millions of years of evolution, nature has developed complex and non-uniform structures that perform extremely well under ambient loading conditions. Mimicking such performance constitutes one of the most ambitious goals in the engineering of artificial composites.

Functionally graded materials (FGMs) provide an artificial approach to reproducing the locally varying compositions encountered in bio-composites, as well as the smooth transitions at the interfaces between their components. Besides exhibiting locally varying mechanical properties, ${ }^{8-10}$ FGMs may combine different and apparently incompatible features within the same structure, giving rise to a variety of unusual, high-impact properties, such as spatially varying electronic performances, ${ }^{11}$ super hardness, ${ }^{12}$ graded refractive indexes, ${ }^{13}$ improved wear resistance and efficient residual stress distribution. ${ }^{14-16}$ Depending on the desired gradient morphology and on the nature of the materials employed, techniques such as chemical vapor deposition (CVD),${ }^{17}$ powder densification,,${ }^{18}$ thermal spraying, ${ }^{19}$ solvent-welding, ${ }^{20}$ centrifugal casting, electrodeposition (EPD) or self-driven transport-based processes ${ }^{21-23}$ may be used. However, these may either be multi-step processes, often requiring the use of computer-aided automated systems and making use of expensive apparatuses and/or complex synthetic strategies, or have strong limitations when parts with complex geometries are required. Moreover, many such processes are inappropriate to soft polymeric materials. An alternative approach is based on transport-based processes arising from magnetic forces, although these have so far received little attention in the literature ${ }^{24}$ The main forces acting on a magnetic particle with radius $r$ immersed in a fluid under the influence of a magnetic field are the magnetic force, $\mathrm{F}^{-} \mathrm{m}$, and the drag force, $\mathrm{F}_{\text {d. }}^{-} \mathrm{F}^{-} \mathrm{m}$ is due to the gradient of the applied magnetic flux density, $\mathrm{B}^{-}$, and the induced magnetic moment $\mathrm{m}^{-}$of the particle (Equation 1), ${ }^{25}$ whereas $\mathrm{F}^{-} \mathrm{d}$ derives from the viscous drag exerted by the suspending medium (with viscosity $\eta$ ) on the moving particle, and is a consequence of the velocity difference, $\Delta \mathrm{v}^{-}$, , between the particle and the fluid (Equation 2). ${ }^{26}$

$$
\begin{aligned}
& \bar{F}_{m}=\frac{1}{\mu_{0}}(\bar{m} \cdot \bar{\nabla}) \bar{B} \\
& \bar{F}_{d}=6 \pi \eta r \Delta \bar{v}
\end{aligned}
$$

As $\mathrm{F}^{-} \mathrm{m}$ and $\mathrm{F}_{\mathrm{d}}^{-}$oppose each other, the particle velocity will increase with the gradient of $\mathrm{B}^{-}$and decrease with $\eta$.

In this study, we investigate the basic principles underlying the motion of superparamagnetic $\mathrm{Fe}_{3} \mathrm{O}_{4} @ \mathrm{SiO}_{2}$ core@shell nanoparticles in an UV-curable hyperbranched polymeric matrix (HBP) under the effect of magnetic field gradients, proposing practical strategies to efficiently increase $\mathrm{F}^{-} \mathrm{m}$ and reduce $\eta$. Analyses of the gradient formation process and of the resulting graded mechanical properties are divided into four sections. First, we characterize the particle concentration gradients by means of SEM and nanoindentation analyses. Second, we focus on the computation of the magnetic force exerted by different arrangements of permanent magnets on $\mathrm{Fe}_{3} \mathrm{O}_{4} @ \mathrm{SiO}_{2}$ nanoparticles and we relate these analytical results with the 
experimentally observed gradient morphologies. Third, we analyze the viscosity of two different classes of uncured nanoparticulate systems based on the HBP matrix and containing either untreated $\mathrm{Fe}_{3} \mathrm{O}_{4} @ \mathrm{SiO}_{2}$ nanoparticles or MPS-functionalized $\mathrm{Fe}_{3} \mathrm{O}_{4} @ \mathrm{SiO}_{2}$ nanoparticles (see Supporting Information for nanoparticle characterization), and we directly relate the observed trends in viscosity to considerations on the effectiveness of the gradient formation process. Finally, the gradients in Young's modulus and in hardness of the synthesized graded materials are assessed by means of nanoindentation tests, after UV-curing.

Such a synthetic procedure efficiently condenses into a one-step process the creation of non-uniform materials, whose design not only enhances the mechanical characteristics, as encountered in some biogenic composites, ${ }^{7}$ but also conveniently limits the use of reinforcing elements to those regions where they are most needed. Although these results are specific to the materials investigated, the underlying synthetic principles have the potential to be applied to many types of polymeric nanocomposites.

\section{Experimental}

\section{Materials}

Iron (III) acetyacetonate ( $\mathrm{Fe}(\mathrm{acac}) 3,99+\%)$ and benzyl ether (99\%) were purchased from Acros. Oleylamine (Tech.70\%), polyoxyethylene(5)nonylphenyl ether (Igepal CO-520), [3-(Methacryloyloxy)propyl] trimethoxysilane (MPS, 98\%), ethanol ( $\geq 99.5$ $\%)$ and the epoxy embedding medium kit were purchased from Aldrich. Cyclohexane (p.A.) was purchased from Applichem. Ammonia solution (25\% min) and tetraethyl orthosilicate (TEOS, $\geq 99 \%$ ) were purchased from VWR and Merck, respectively. The hyperbranched polyester acrylated oligomer (HBP, commercial name CN2302) was purchased from Sartomer, whereas the photo-initiator (Lucirin TPO) was purchased from BASF. All products were used as received without any further purification. Permanent magnets were block NdFeB (Neodymium Iron Boron-N42 grade) magnets purchased from Supermagnete. The structure holding the two permanent magnets in repulsion was made in austenitic steel.

\section{Synthesis of bare and coated $\mathrm{Fe}_{3} \mathrm{O}_{4}$ nanoparticles}

The synthetic procedure for $\mathrm{Fe}_{3} \mathrm{O}_{4}$ nanoparticles and $\mathrm{Fe}_{3} \mathrm{O}_{4} @$ silica core-shell nanoparticles has been reported in a previous publication. ${ }^{27}$ MPS-functionalized coreshell nanoparticles were synthesized analogously to non-functionalized ones, with the only difference that $24 \mathrm{~h}$ after the addition of TEOS, 3-(Methacryloxy)propyltrimethoxysilane was added into the reaction flask in a quantity around 1/14 of the TEOS amount.

\section{Preparation of nanocomposites}

As described earlier, ${ }^{27}$ the photo-initiator was first dissolved in the HBP in a $6 \mathrm{wt} \%$ fraction and subsequently the selected amount of $\mathrm{Fe}_{3} \mathrm{O}_{4}$ or core-shell nanoparticles was added. A certain amount of as-prepared nanocomposites were placed between two UV-transparent polystyrene sheets $(16 \mathrm{~mm} \times 16 \mathrm{~mm} \times 0.3 \mathrm{~mm}$, made by hot pressing polystyrene beads) kept at a distance of $150 \mu \mathrm{m}$ by two plastic spacers (16 
$\mathrm{mm} \times 3 \mathrm{~mm} \times 0.15 \mathrm{~mm}$ ) which ensured a constant thickness of the final polymeric films. The polymerization process was performed irradiating the samples from both sides using two identical 200W mercury bulb UV lamps (OmniCure 2000, Exfo, Canada). Using a calibrated radiometer (Silver Line, CON-TROL-CURE, Germany), the measured UV-A light intensity on the sample surface ensured by each lamp was around $190 \mathrm{~mW} \mathrm{~cm}^{-2}$. Small variations in the final thickness of the films were not excluded due to differences in mechanical properties and polymerization shrinkage. To analyze the nanoparticles distribution before and after the application of the magnetic field, the films were first embedded in an epoxy medium in order to guarantee their dimensional stability and subsequently cut with a diamond wire cutting machine to expose their cross-sectional area.

\section{Methods}

Infrared spectrometry was performed using a Perkin Elmer Spectrum One MIR (600$4000 \mathrm{~cm}^{-1}$ ) with an ATR accessory.

Embedded samples were cut using a Well Diamond Wire Saw, polished with a Struers Dap-V polishing machine and made conductive for SEM analysis upon deposition of a $20 \mathrm{~nm}$ carbon layer with a Cressington 108 carbon/a carbon-coater. The concentration gradient was investigated analyzing the Si content by SEM-EDX analysis using a FEI XLF-30 FEG at an accelerating voltage $\mathrm{V}_{\text {acc }}$ of $13 \mathrm{kV}$ and at a constant working distance $(11 \mathrm{~mm})$ and spot size (4). An average elemental composition of the samples was obtained by data collection over a $150 \mu \mathrm{m}$ x $120 \mu \mathrm{m}$ sized window.

Nanoindentation measurements were performed on the transverse cross-sectioned samples using a Nano Indenter XPTM (MTS Nano Instruments) with a three-sided diamond Berkovich indenter. Different series of tests were conducted with and without continuous stiffness measurements (CSM) mode. Under CSM engagement, indenter was operated at the tip frequency of $45 \mathrm{~Hz}$, harmonic displacement of $2 \mathrm{~nm}$, and constant nominal strain rate of $0.05 \mathrm{~s}^{-1}$. For tests with the CSM mode off, multiple steps of cyclic loading approach were applied to capture load-displacement hysteresis due to viscoelastic effects. For all tests the indenter tip approached the surface from a distance of $1000 \mathrm{~nm}$ at a rate of $10 \mathrm{~nm} / \mathrm{s}$, and the maximum penetration depth into the sample was set to $1000 \mathrm{~nm}$. Hardness and Young's modulus were determined using the Oliver and Pharr's analysis method ${ }^{28}$ and assuming a Poisson's ratio of 0.35 . To provide a simple comparison, the reported values of hardness and Young's modulus correspond to a penetration depth of $1000 \mathrm{~nm}$ into the samples.

\section{Results and discussion}

\section{Graded Morphologies}

In order to facilitate the comparison between different samples and testing conditions, all the SEM-EDX images showing the results of the synthetic process are summarized in a single table (Figure 1). Each panel shows the Si content along the cross-section of approximately $150 \mu \mathrm{m}$ thick nanoparticulate UV-cured films based on the acrylated hyperbranched polymeric matrix (HBP). Prior to curing, polymeric films containing 1 vol\% or 8 vol\% of homogeneously distributed $\mathrm{Fe}_{3} \mathrm{O}_{4} @ \mathrm{SiO}_{2}$ nanoparticles (Figure 1 
a1-f1) were placed under the effect of the magnetic field gradient generated either by a single magnet or by two magnets in repulsion configuration (i.e. with the magnetizations opposing each other). When only one magnet was employed, the distance $z$ from its surface to the closest edge of the film was chosen in order to maximize the $\mathrm{z}$-component of $\mathrm{F}^{-} \mathrm{m}$ (Figure $2 \mathrm{a}$ ) and accelerate the gradient formation process along the z-direction (see Section 2.2). Similarly, when two magnets in repulsion configuration were used, their relative distance $d$ as well as the distance $z$ between one of them and the closest edge of the nanoparticulate film (Figure $2 b$ ) were optimized for the maximization of the z-component of F, $\mathrm{m}$ (see Section 2.2).

The gradient morphology was then observed after 1 hour (Figure 1 a2-f2) and 24 hours (Figure $1 \mathrm{a} 3-\mathrm{f3}$ ) from the initial application of $\mathrm{F}^{-} \mathrm{m}$. Even though the size of the synthesized samples ( $1 \mathrm{~cm} \times 1 \mathrm{~cm} \times 150 \mu \mathrm{m}$, see Supporting Information) is limited by the employed lab-scale set up, it is believed that bigger sample sizes could be easily attained in industrial applications, for which the utilization of stronger magnets would not be precluded. While not being able to achieve the same grading capability of layer-by-layer techniques, ${ }^{20}$ which although require a more costly and time consuming preparation, it is evident that the proposed technique enables the creation of rather steep concentration profiles within reasonable amounts of time. Moreover, it should be pointed out that the proposed process makes use of nanofillers that, being 23 orders of magnitude smaller than those found in the great majority of graded polymeric composites synthesized through different field-based processes (e.g. centrifugal casting ${ }^{29}$ ), are intrinsically affected by a rather weak driving force. 


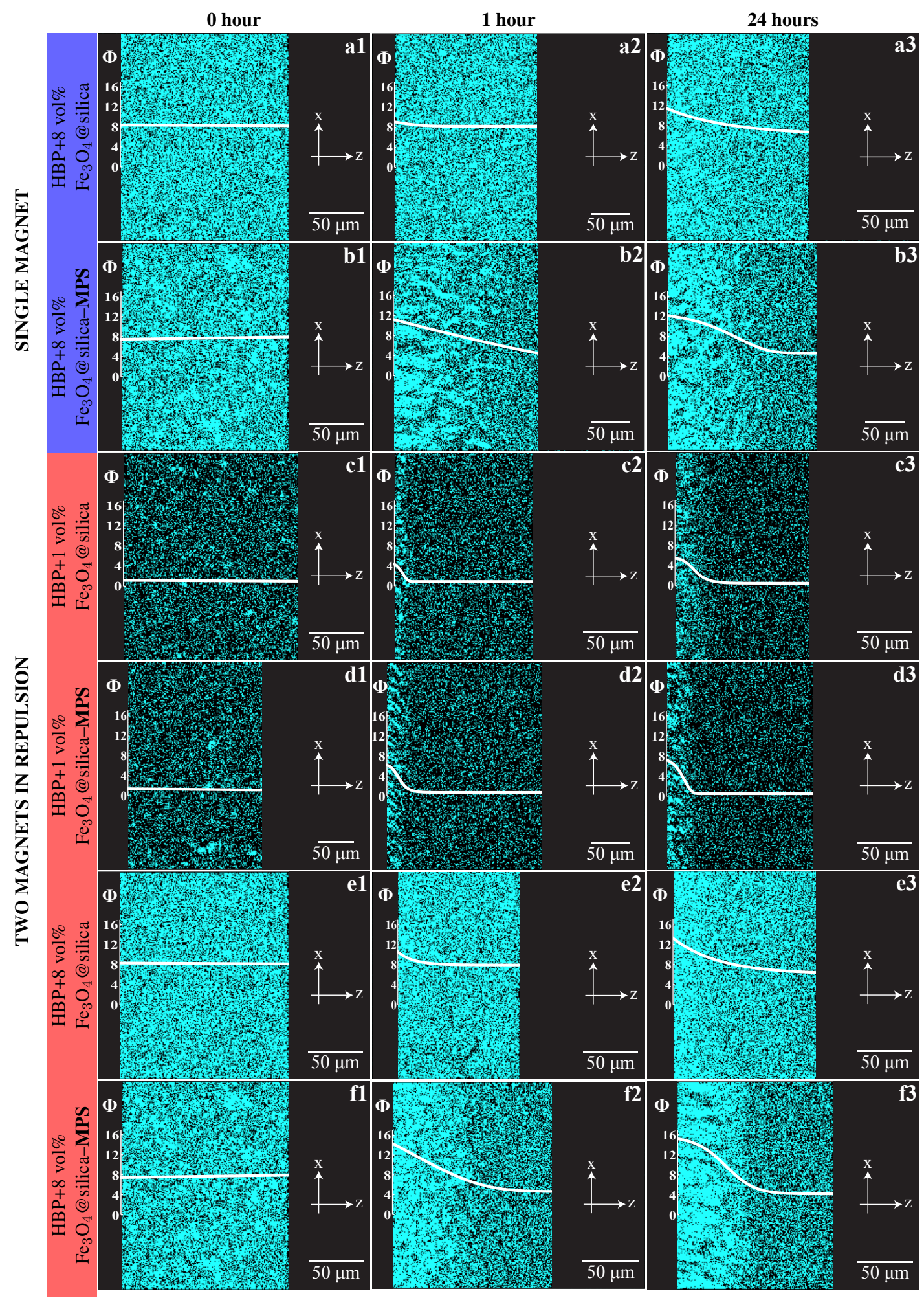

Figure 1. Cross-sectional SEM-EDX spectral images showing the Si content of different samples (composition indicated on the left side of the table). Samples have been under the influence of the magnetic force generated by a single magnet (blue) and two magnets in repulsion configuration (red) for the time indicated at the top of the table. The superimposed curves indicate the nanoparticle volume fractions $\Phi(\times 100)$ all along the sample cross-sections. 


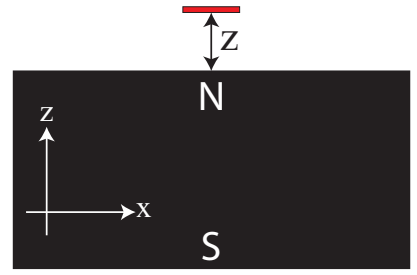

(a)

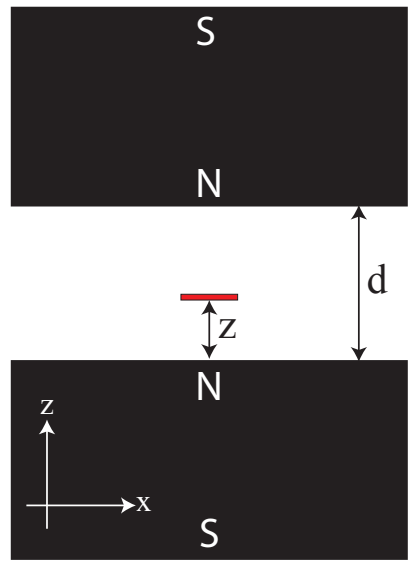

(b)

Figure 2. Schematic representation of the nanoparticulate film (red) placed at a distance z from a single magnet (a) and from one of two facing magnets (b). In (b) the two magnets are positioned at a distance d between each other.

\section{Magnetic force}

This section analyses the influence of the magnetic force intensity on the gradient formation process. In particular, we compute the magnetic forces exerted by three different arrangements of permanent magnets on the utilized superparamagnetic $\mathrm{Fe}_{3} \mathrm{O}_{4} @ \mathrm{SiO}_{2}$ nanoparticles, which have been previously morphologically and magnetically characterized. ${ }^{27}$ The first case describes a single magnet with a positive magnetization; the second one considers two magnets in repulsive configuration (the top magnet having negative magnetization and the other a positive one), whereas the third involves two magnets in attractive configuration (both with a positive magnetization). All the values for the magnetic force reported in the following pertain to a single $\mathrm{Fe}_{3} \mathrm{O}_{4}$ nanoparticle (with radius $=2.85 \mathrm{~nm}$ ), which constitute the magnetic domain of the core-shell particles (with radius $=12.8 \mathrm{~nm}$ ) employed as fillers. As can be seen in Figure 3, the highest values of the z-component of $\mathrm{F}^{-} \mathrm{m}$ acting on a $\mathrm{Fe}_{3} \mathrm{O}_{4}$ nanoparticle are encountered when two magnets in repulsion configuration are employed and, specifically for the studied materials, when $\mathrm{d}=1.6 \mathrm{~cm}$ and $\mathrm{z}=0 \mathrm{~cm}$ (Table 1). The corresponding highest values of the $\mathrm{z}$-component of $\mathrm{F}^{-} \mathrm{m}$ are encountered at $\mathrm{z}=2 \mathrm{~mm}$ for the single magnet case, whereas at $\mathrm{d} \geq 80 \mathrm{~mm}$ and $\mathrm{z}=0 \mathrm{~mm}$ for the case of two magnets in attraction configuration. In particular, the highest value of the $\mathrm{z}$-component of $\mathrm{F}^{-} \mathrm{m}$ generated by two magnets in repulsion exceeds by $24 \%$ the maximum force generated either by a single magnet or by two magnets in attraction configuration. The method for obtaining these values is discussed in greater details in the Supporting Information. 


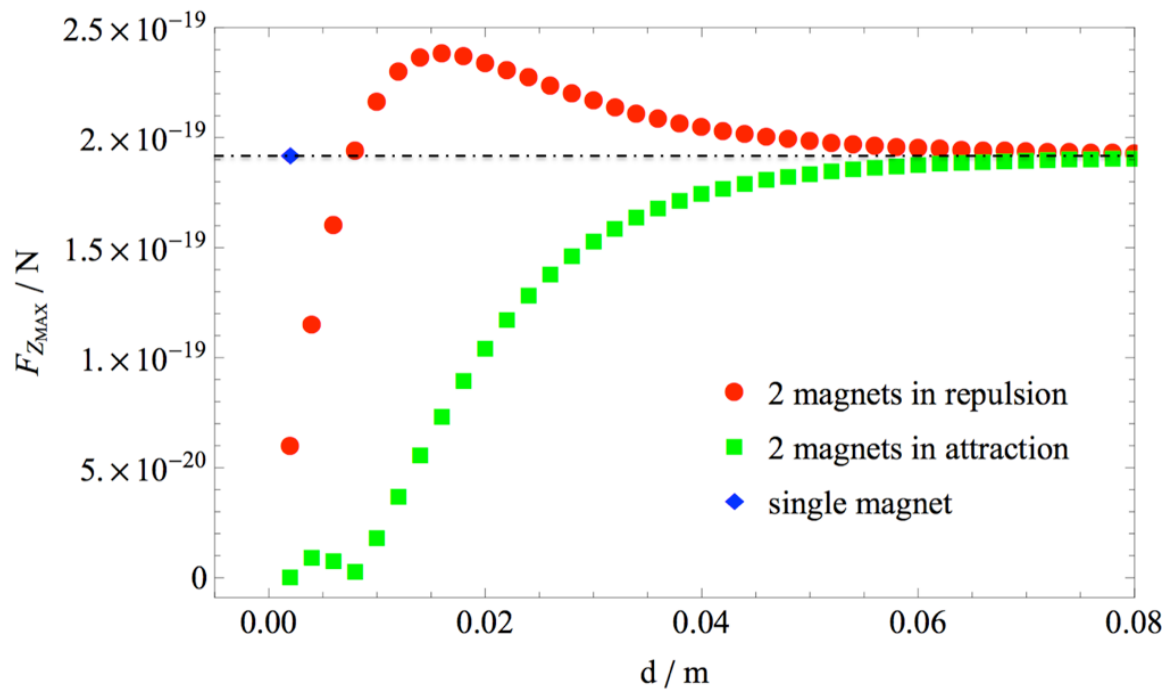

Figure 3. Trend of the highest values of the z-component of the magnetic force $\mathrm{F}^{-} \mathrm{m}$ exerted on a single $\mathrm{Fe}_{3} \mathrm{O}_{4}$ nanoparticle as a function of the distance $d$ between the magnets ( $z=2 \mathrm{~mm}$ for the single magnet case, $z=0 \mathrm{~mm}$ for both the 'two magnets' configurations).

Table 1. Highest computed z-component of the magnetic force acting on a single $\mathrm{Fe}_{3} \mathrm{O}_{4}$ nanoparticle with corresponding values of the parameters $d$ and $z$ for three different configurations of permanent magnets.

\begin{tabular}{llll}
\hline CONFIGURATION & $\boldsymbol{d} / \mathbf{m m}$ & $\boldsymbol{z} / \mathbf{m m}$ & $\mathbf{F}_{\mathbf{m}}^{-} / \mathbf{N}$ \\
\hline Single magnet & - & 2 & $1.914 \cdot 10^{-19}$ \\
2 magnets in repulsion & 16 & 0 & $2.378 \cdot 10^{-19}$ \\
2 magnets in attraction & $\geq 80$ & 0 & $1.914 \cdot 10^{-19}$ \\
\hline
\end{tabular}

Following the results of the computational studies, only the effects of the magnetic forces generated by a single magnet and by two magnets in repulsion configuration were further investigated. When the polymeric matrix is filled with $8 \mathrm{vol} \%$ of untreated $\mathrm{Fe}_{3} \mathrm{O}_{4} @$ silica nanoparticles (HBP+8 vol\% $\mathrm{Fe}_{3} \mathrm{O}_{4} @$ silica), the composition remains almost unchanged after 1 hour under the effect of the field generated by the single magnet (Figure 4a-1 hr), whereas a gradient is already noticeable when two magnets in repulsion are employed (Figure $4 \mathrm{~b}-1 \mathrm{hr}$ ). After 24 hours, a continuous compositional gradient is developed for both cases (Figure 4a and $4 \mathrm{~b} 24 \mathrm{hr}$ ), but ranging from 13 to $6 \mathrm{vol} \%$ if two magnets in repulsion are used and from 11.5 to 6.5 vol\% when only one magnet is used. In order to be able to compare the developments of the compositional gradient between the various samples, the curves showing the particle volume fraction as a function of the position $z$ were extrapolated to a fixed length of $150 \mu \mathrm{m}$ and the average distance traveled (ADT) by the nanoparticles was defined as

$A D T=\frac{\sum_{i=1}^{150} \Phi_{i}(t) \times i}{\sum_{i=1}^{150} \Phi_{i}(t)}-\frac{\sum_{i=1}^{150} \Phi_{i}(0) \times i}{\sum_{i=1}^{150} \Phi_{i}(0)}$

where $\Phi_{i}$ is the volume fraction of nanoparticles at the position $i$ along the thickness of the sample and the subscripts ( $\mathrm{t}$ and 0 ) represent the time spent under the influence of the external magnetic field. The as-calculated ADT points were fitted with a 
negative exponential function of the type $y=a(1-\exp (-k x))$. Making use of this definition, we can see (Figure 4c) that the gradient created in 24 hours with a single magnet can be generated with two magnets in repulsion within half of the time. In general, for both systems the gradient undergoes a continuous development during the whole 24 hours, but turns out to be significantly faster when two magnets in repulsion are used.
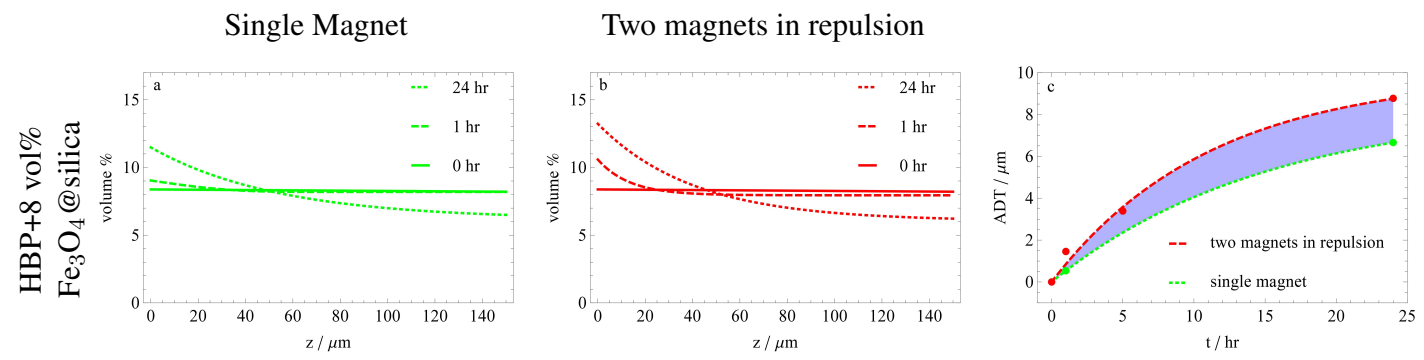

Figure 4. (a,b) Concentration profiles of $\mathrm{Fe}_{3} \mathrm{O}_{4} @$ @ilica nanoparticles as a function of the position along the sample cross-section for the single magnet case and the case of two magnets in repulsion configuration, evaluated at different times. (c) Calculated ADT (Eq.3) as a function of the time spent under the influence of the externally applied magnetic field (the films span the range from $z=0$ to $z=150 \mu \mathrm{m})$.

As a matter of fact, the magnitude of the applied magnetic force is directly reflected both on the steepness of the maximum achievable gradient and on the speed of the gradient formation process. The magnetic force increment generated by a simple setup of permanent magnets placed in repulsion configurations can not only reduce the time needed to attain a certain gradient, but can effectively induce nanoparticle motion even at higher local viscosities, giving rise to gradients unattainable with the employment of a single magnet.

\section{Drag force}

This section investigates the effect of the viscosity of the nanoparticulate suspensions on the gradient development process. In particular, two systems filled with different amounts of non-functionalized and MPS-functionalized $\mathrm{Fe}_{3} \mathrm{O}_{4} @$ silica nanoparticles are studied and compared. Ruckenstein et al. already clarified the importance of surface treatment on the dispersing ability of nanoparticles in polymeric media. ${ }^{30,31}$ Here, rheological tests (Figure 5) show that for all the studied systems acrylation of the particles surface with MPS has a significant effect on the viscoelastic properties of the composite prior to UV-curing. Following the trend previously reported by Geiser et al., ${ }^{32}$ systems containing acrylated nanoparticles always evidence a much lower complex viscosity than that of the suspensions filled with non-functionalized particles (Figure 5), due to a strong reduction of elastic effects ( $\eta$ "). This is most likely due to H-bonding suppression and to lower particle-polymer interactions. In particular, the complex viscosity and elastic contribution of the system filled with $8 \mathrm{vol} \%$ of $\mathrm{Fe}_{3} \mathrm{O}_{4} @$ silica-MPS nanoparticles are, at $\omega=0.1 \mathrm{rad} / \mathrm{s}, 6$ and 40 times lower, respectively, than those of the suspension containing the same amount of nonacrylated particles. 


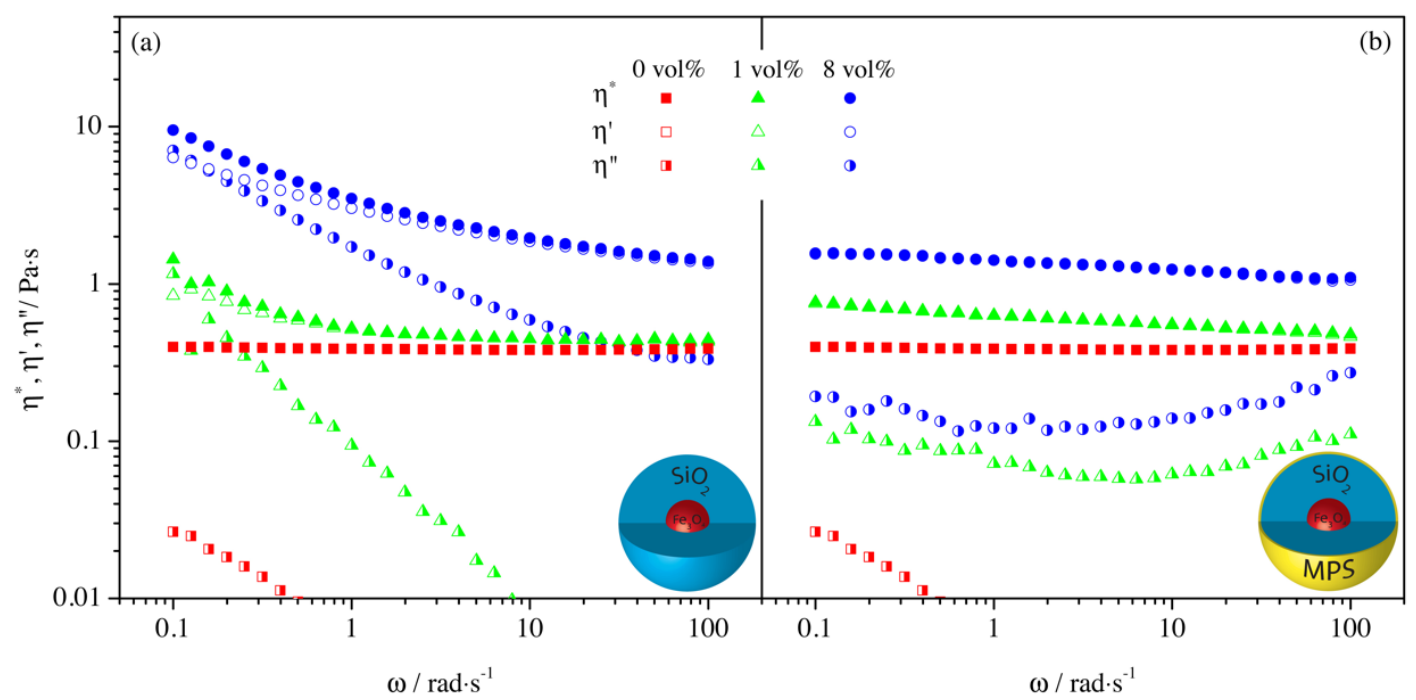

Figure 5. Complex viscosity, $\eta^{*}$, and viscous and elastic contribution, $\eta$ ' and $\eta^{\prime \prime}$, respectively, of the nanoparticulate-composites filled with non-functionalized $\mathrm{Fe}_{3} \mathrm{O}_{4} @$ silica nanoparticles (a) and with MPS-functionalized $\mathrm{Fe}_{3} \mathrm{O}_{4} @$ silica nanoparticles (b) as a function of frequency and loading level.

In order to compare the effect of different drag forces we investigated the development of the concentration gradient, under the influence of the same magnetic field generated by two magnets in repulsion configuration, for the two systems containing non-functionalized and MPS-functionalized $\mathrm{Fe}_{3} \mathrm{O}_{4} @$ silica nanoparticles. Suspensions containing 1 vol\% of non-functionalized nanoparticles (Figure 6a1) developed a gradient spanning from 4.5 to 0.8 vol\% after 1 hour and from 5.5 to 0.2 vol $\%$ after 24 hours. The small decrease in viscosity characterizing the systems filled with 1 vol\% of $\mathrm{Fe}_{3} \mathrm{O}_{4} @$ silica-MPS nanoparticles (Figure 6b1) compared to those filled with non-silanized particles was sufficient to induce a faster process, enabling the formation of a gradient ranging from 6 to 0.7 vol\% after 1 hour and from 7.2 to 0.2 vol $\%$ after 24 hours.

It should be pointed out that during the magnetophoretic process the characteristic velocities of $\mathrm{Fe}_{3} \mathrm{O}_{4} @$ silica particles, covering distances in the order of some tens of microns in time frames in the order of hours, can be estimated to be at least one order of magnitude smaller than those experienced by the nanoparticles in the rheological test. In light of this, it is reasonable to assume that a more precise evaluation of the viscosity experienced by the magnetic nanofiller during the magnetophoretic process should be done at angular frequencies lower than $0.1 \mathrm{rad} / \mathrm{s}$, which is however the limit imposed by the instrument. At such low values of $\omega$, given that nanocomposites containing MPS-functionalized nanoparticles exhibit a slight shear thinning behavior, whereas systems containing non-functionalized $\mathrm{Fe}_{3} \mathrm{O}_{4} @$ silica nanoparticles evidence a non-linear decrease of the complex viscosity with increasing applied angular frequency, the difference in $\eta^{*}$ would most likely be much higher than that evidenced at $0.1 \mathrm{rad} / \mathrm{s}$. This would explain why MPS surface treatment has such an effect on the speed of the magnetophoretic process and why, even at particle volume fractions as low as $1 \%$, the gradient formation process (Figure $6 \mathrm{c} 1$ ) reaches a steady state in more than 10 hours for systems containing non-functionalized particles and within 3 hours for those filled with MPS-treated ones. 

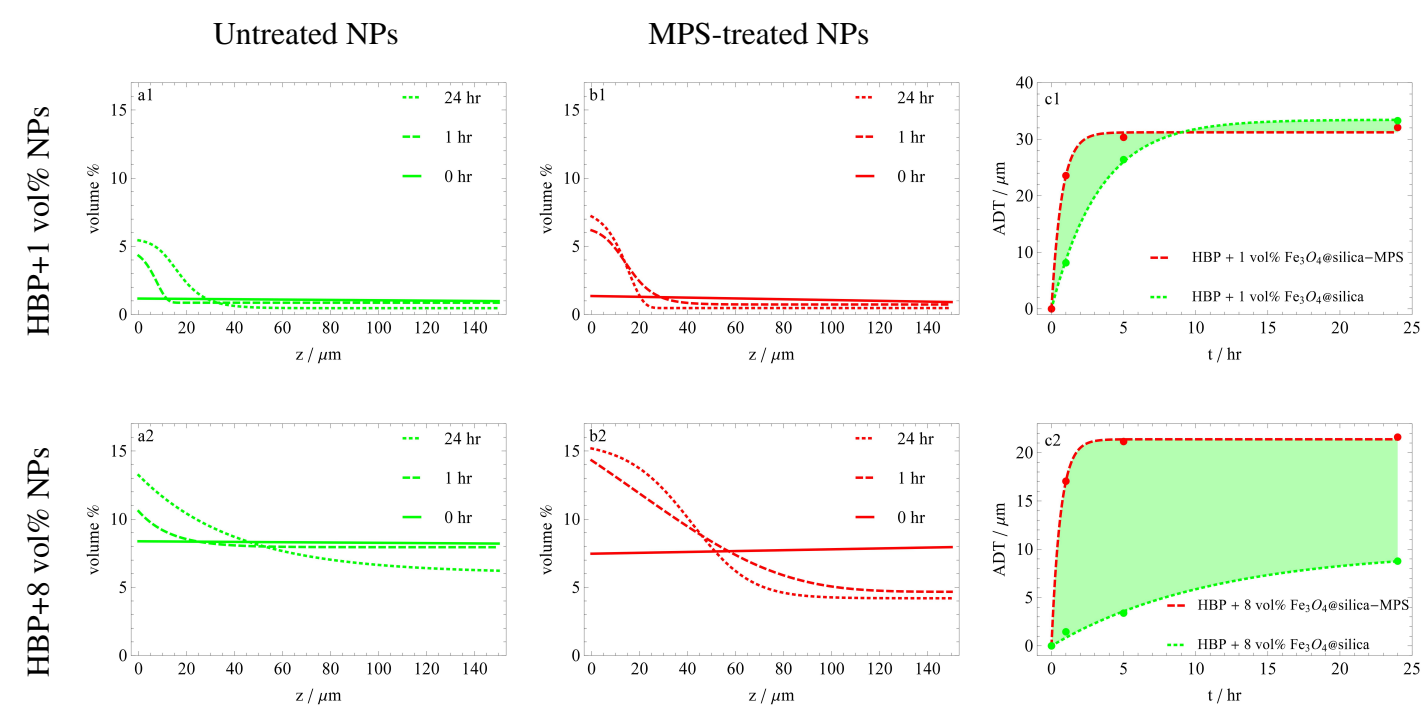

Figure 6. Concentration profiles of non-functionalized (a) and MPS-functionalized (b) $\mathrm{Fe}_{3} \mathrm{O}_{4} @$ silica nanoparticles as a function of the position along the sample cross-section. Samples have been under the influence of the magnetic field gradient generated by two magnets placed in repulsion configuration at a distance $d=1.6 \mathrm{~cm}$ (c). Calculated ADT (Eq.3) as a function of the time spent under the influence of the externally applied magnetic field.

Upon going towards higher loading levels the different behaviors of the suspensions filled with untreated and MPS-treated nanoparticles are much more pronounced. When HBP is loaded with 8 vol\% of non-functionalized particles (Figure 6a2), the nanoparticle distribution remains almost unchanged after 1 hour under the effect of the magnetic field, whereas a very mild concentration gradient, ranging from 13 to 6 vol $\%$, is generated after 24 hours. On the contrary, when the HBP is loaded with 8 vol\% of $\mathrm{Fe}_{3} \mathrm{O}_{4} @$ silica-MPS nanoparticles (Figure 6b2), the gradient already spans from 14.5 to 4.5 vol\% after 1 hour and from 15 to 4 vol\% after 24 hours. Moreover, a steady state is reached after 10 hours for the suspensions containing $8 \mathrm{vol} \%$ of nonfunctionalized particles, whereas the gradient formation process is almost complete within 1 hour if $\mathrm{Fe}_{3} \mathrm{O}_{4} @$ silica-MPS nanoparticles are employed (Figure 6c2). As a matter of fact, the viscosity control through particle surface treatment turns out to be a fundamental aspect to take into account for a fast gradient development process, becoming more and more important upon going towards high particle loading fractions.

Finally, a good correlation between the average velocity $\left(v_{\exp }\right)$ of the particle calculated from their average distance travelled (ADT, Equation 3) during the whole 24 hours and the velocity $\left(\mathrm{v}_{\text {calc }}\right)$ deduced from the analytical equations describing $\mathrm{F}^{-} \mathrm{m}$ (Equation 1) and $\mathrm{F}_{\mathrm{f}}^{-}$(Equation 2) was obtained, in spite of simplified hypotheses (Table 2). For the evaluation of $\mathrm{F}^{-}$, the viscous viscosity $\eta^{\prime}$ ' was inserted in Equation 2. The difference between experimental and calculated values is attributed to the viscoelastic nature of the fluid, which leads the expression for the viscous drag force to depart from the classical result (Equation 2), ${ }^{33}$ and to other effects such as aggregation and dipole interactions. ${ }^{34}$ 
Table 2. Experimental velocities (vexp) of the different nanofillers as calculated from their ADT (Eq.3) during 24 hours and their velocities $\left(v_{\text {calc }}\right.$ ) calculated starting from the analytical expressions for $F_{,}^{-} m$ and $F_{,}^{-} f$. The viscous viscosities $\eta$ ' of the different systems are also indicated.

\begin{tabular}{lllllll}
\hline$\Phi$ & 0.01 & 0.01 & 0.08 & 0.08 & 0.08 & 0.08 \\
\hline Surface & - & $\mathrm{MPS}$ & - & - & $\mathrm{MPS}$ & $\mathrm{MPS}$ \\
Configuration & $2 \mathrm{M}^{\mathrm{a}}$ & $2 \mathrm{M}^{\mathrm{a}}$ & $1 \mathrm{M}^{\mathrm{b}}$ & $2 \mathrm{M}^{\mathrm{a}}$ & $1 \mathrm{M}^{\mathrm{b}}$ & $2 \mathrm{M}^{\mathrm{a}}$ \\
$\mathbf{\eta}^{\prime}(\mathbf{P a} \cdot \mathbf{s})$ & 0.93 & 0.75 & 6.38 & 6.38 & 1.55 & 1.55 \\
$\mathbf{v}_{\text {exp }}(\boldsymbol{\mu m} / \mathbf{h})$ & 1.41 & 1.39 & 0.28 & 0.36 & 0.61 & 0.96 \\
$\mathbf{v}_{\text {calc }}(\boldsymbol{\mu m} / \mathbf{h})$ & 1.91 & 2.36 & 0.22 & 0.28 & 0.92 & 1.14 \\
\hline
\end{tabular}

a) Two magnets in repulsion configuration; ${ }^{\text {b) }}$ Single magnet configuration

\section{Graded Young's modulus and hardness}

To correlate the local particle volume fraction with the corresponding mechanical properties, indentations were performed all along the cross-section of the $\approx 150 \mu \mathrm{m}$ thick samples (Figure 7). In light of the better results obtained during the gradient formation process, only the samples filled with MPS-treated nanoparticles were tested.
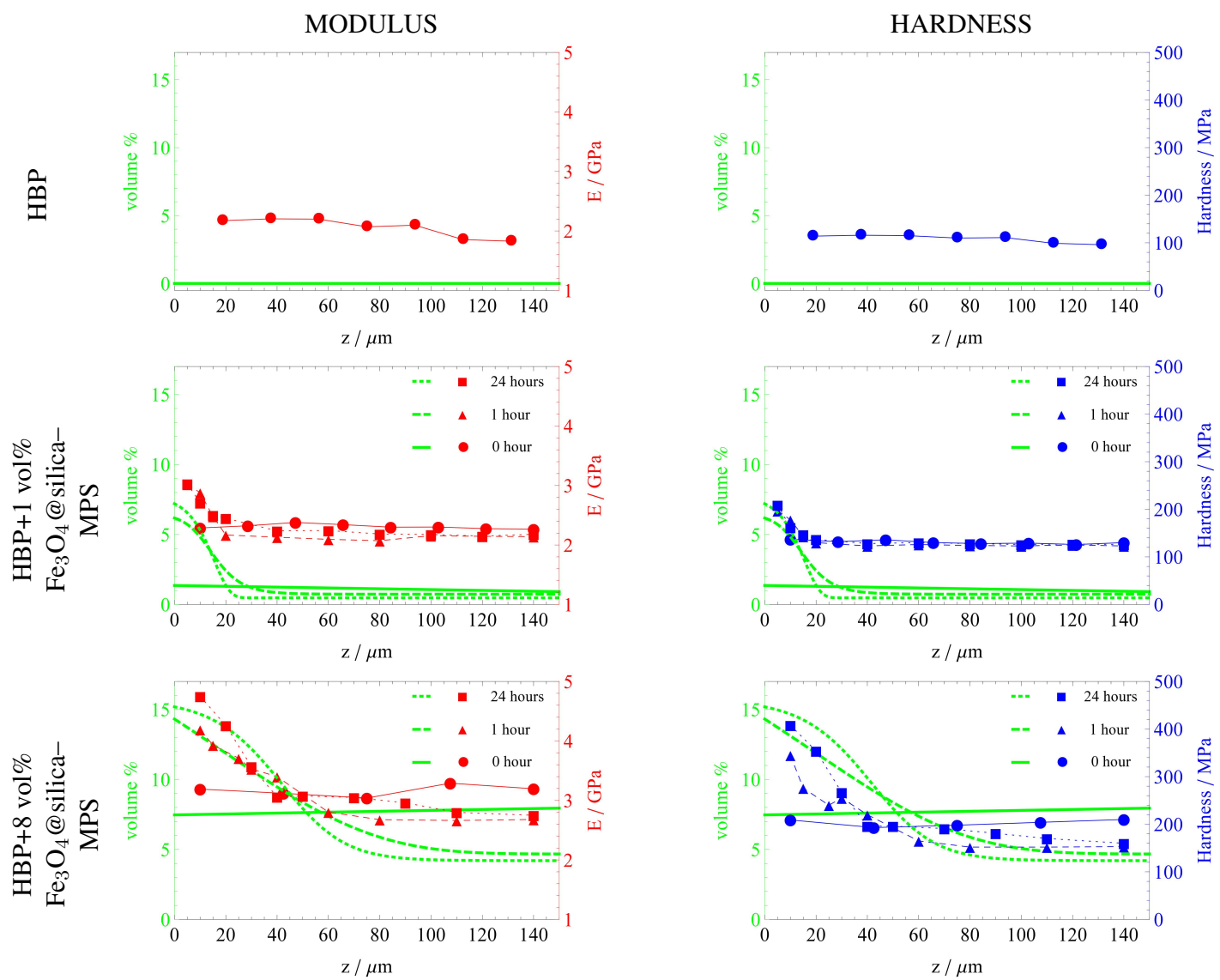

Figure 7. Elastic modulus and hardness as a function of the position $z$ along the film thickness for the formulations containing MPS-treated NPs prior to ( $\boldsymbol{\bullet}$ ) and after 1 ( $\boldsymbol{\Delta})$ and 24 hours $(\boldsymbol{\square})$ from the application of the magnetic field gradient generated by two magnets in repulsion configuration.

Increments in Young's modulus and hardness with respect to the pure HBP $(\mathrm{E}=2.06$ $\mathrm{GPa}, \mathrm{H}=109 \mathrm{MPa}$ ) are already noticeable when 1 vol\% of $\mathrm{Fe}_{3} \mathrm{O}_{4} @ \mathrm{SiO}_{2}-\mathrm{MPS}$ nanoparticles are homogeneously dispersed in the polymeric matrix $(\mathrm{E}=2.31 \mathrm{GPa}, \mathrm{H}$ $=131 \mathrm{MPa}$ ), becoming more evident when 8 vol $\%$ of $\mathrm{Fe}_{3} \mathrm{O}_{4} @ \mathrm{SiO}_{2}-\mathrm{MPS}$ nanoparticles are used $(\mathrm{E}=3.17 \mathrm{GPa}, \mathrm{H}=203 \mathrm{MPa})$. 
When uncured nanosuspensions filled with 1 vol\% of homogeneously distributed $\mathrm{Fe}_{3} \mathrm{O}_{4} @ \mathrm{SiO}_{2}$-MPS nanoparticles are left under the effect of the magnetic field gradient generated by two magnets in repulsion configuration, remarkable variations in elastic modulus $(+41 \%)$ and hardness $(+60 \%)$ when going from particle-depleted to particle-enriched regions of the cured composites are noticeable already after 1 hour. Remaining the gradient morphology almost unaltered (Figure 6-b1), these variations remain almost unchanged ( $+39 \%$ in modulus and $+65 \%$ in hardness) after 24 hours from the initial application of the magnetic field gradient. Variations of mechanical properties become more evident when going towards higher particle volume fractions. As a matter of fact, outstanding gradations in elastic modulus (up to $+56 \%$ ) and hardness (up to $+125 \%$ ) are encountered when moving from particledepleted to particle-enriched zones in films filled with 8 vol\% of $\mathrm{Fe}_{3} \mathrm{O}_{4} @ \mathrm{SiO}_{2}-\mathrm{MPS}$ nanoparticles and having been under the effect of the magnetic field gradient for just 1 hour. Following the development of the gradient morphology (Figure 6-b2), these variations become even more important after 24 hours of magnetic field gradient application, reaching values of $+70 \%$ for the modulus and $+152 \%$ for the hardness. Not only the synthesized graded materials evidence considerable variations in mechanical properties within their structure, but they also show regions characterized by much higher moduli and hardness compared to the corresponding homogeneous counterparts (Figure 8). In particular, 1 hour of magnetic field gradient application gives rise to a substantial local increase in modulus (by 31\%) and hardness (by 50\%) compared to the corresponding values of the nanocomposites containing $1 \mathrm{vol} \%$ of homogeneously distributed $\mathrm{Fe}_{3} \mathrm{O}_{4} @ \mathrm{SiO}_{2}$-MPS nanoparticles. While the increment in elastic modulus remains unchanged after 24 hours of magnetic field gradient application $(+31 \%)$, hardness reaches a value $59 \%$ greater than that of the homogeneous nanocomposite. When nanosuspensions filled with $8 \mathrm{vol} \%$ of homogeneously distributed $\mathrm{Fe}_{3} \mathrm{O}_{4} @ \mathrm{SiO}_{2}$-MPS nanoparticles are left under the effect of the magnetic field, the increments in elastic modulus and hardness compared to the corresponding homogeneous samples are in the order of $32 \%$ and $70 \%$, respectively, after 1 hour, and of $50 \%$ and 101\%, respectively, after 24 hour. As a matter of fact, the local concentration of particles within a specific region of the composite not only gives rises to continuously graded materials presenting substantial variations in elastic modulus and hardness within their body and characterized by smooth transitions between mechanically different areas, but also represents an efficient strategy to rationalize the distribution of reinforcing elements, locally maximizing the stiffness and the hardness in nanocomposites. 

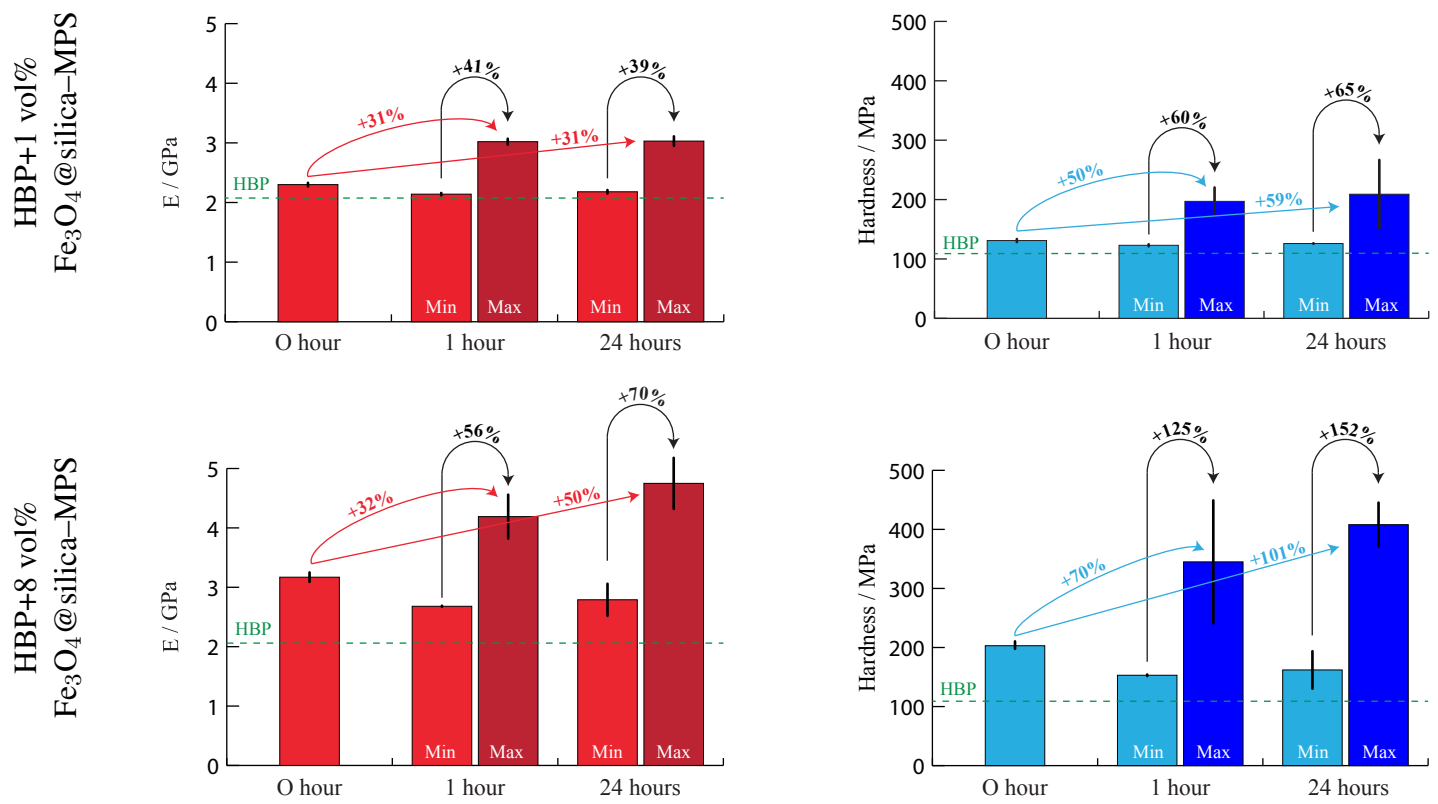

Figure 8. Histograms showing the enhancement of elastic moduli and hardness of the cured nanocomposites filled with 1 vol\% and 8 vol\% of $\mathrm{Fe}_{3} \mathrm{O}_{4} @ \mathrm{SiO}_{2}-\mathrm{MPS}$ nanoparticles. For the samples which have been under the effect of the magnetic field gradient, the minimum and maximum elastic moduli and hardness (i.e. corresponding to the values at the opposing edges of the film) are displayed. The green dashed line refers to the pure $H B P$.

Even though it is not the goal of this work to deepen the analysis on the mechanics of the synthesized nanocomposites, useful informations can still be deduced upon application of appropriate model describing the experimental behavior of heterogeneous materials. Upper and lower bounds models for the elastic modulus of composites are usually described by means of the classical rule of mixtures (RoM, Equation 4) and the inverse rule of mixtures (Equation 5)

$$
\begin{aligned}
& E=\Phi E_{N P}+(1-\Phi) E_{H B P} \\
& E=\left[\Phi / E_{N P}+(1-\Phi) / E_{H B P}\right]^{-1}
\end{aligned}
$$

where $\mathrm{E}, \mathrm{E}_{\mathrm{NP}}$ and $\mathrm{E}_{\mathrm{HBP}}$ represent the values for the elastic moduli of the composite, of $\mathrm{Fe}_{3} \mathrm{O}_{4} @ \mathrm{SiO}_{2}-\mathrm{MPS}$ nanoparticles and of HBP, respectively, and $\Phi$ is the volume fraction of core-shell particles. The elastic modulus of $\mathrm{Fe}_{3} \mathrm{O}_{4} @ \mathrm{SiO}_{2}-\mathrm{MPS}$ nanoparticles was assumed to be the same as that of $\mathrm{SiO}_{2}$ nanoparticles produced via sol-gel method, and was taken equal to $20 \mathrm{GPa}$ (Zhang et al. ${ }^{35}$ reported a value of 18 GPa). The Halpin-Tsai model (Equation 6) ${ }^{36,37}$ is also generally applied to random or nearly random distributions of reinforcements arranged in a matrix, and takes the form:

$E=E_{H B P} \frac{1+\xi \beta \Phi}{1-\beta \Phi}$

where $\beta$ is 


$$
\beta=\frac{E_{N P} / E_{H B P}-1}{E_{N P} / E_{H B P}+\xi}
$$

and $\xi$ is a 'structure' parameter, accounting for the filler geometry and aspect ratio. The fact that experimental data are well fitted by the classical rule of mixtures, which does not take into account the discontinuous nature of the particle assembly and the particle-matrix interplay, indicates a strong interaction between particles and dispersing matrix. The best fitting using the Halpin-Tsai model (Figure 9a) was obtained with $\xi=34$, indicating that, most likely due to interparticle magnetic attractions, the nanoparticles give rise to distributions with high aspect ratios ${ }^{38}$ aligning themselves along preferential directions (i.e. the direction of the magnetic field gradient).

As for the elastic modulus, upper and lower bounds models describing the hardness of the synthesized nanocomposites can be represented by the classical rule of mixtures (RoM, Equation 8$)^{39}$ and the inverse rule of mixtures (Equation 9):

$$
\begin{aligned}
& H=\Phi H_{N P}+(1-\Phi) H_{H B P} \\
& H=\left[\Phi / H_{N P}+(1-\Phi) / H_{H B P}\right]^{-1}
\end{aligned}
$$

where $\mathrm{H}, \mathrm{H}_{\mathrm{NP}}$ and $\mathrm{H}_{\mathrm{HBP}}$ represent the hardness values of the composite, of core-shell particles (taken equal to that of silica, i.e. $2800 \mathrm{MPa})^{40}$ and of HBP, respectively, and $\Phi$ is the volume fraction of core-shell particles. In order to account for the discontinuous nature of the reinforcing phase, in the present work a yielding model for the hardness was used ${ }^{41}$

$$
H=H_{H B P} \frac{1-\Phi}{1+A \Phi} \exp [B \Phi]
$$

where the fractional term in the right-hand-side describes the change of effective particle cross-section as a function of particle fraction $\Phi, \mathrm{B}$ is an interface interaction parameter and $\mathrm{A}$ is a shape parameter associated with packing features of reinforcing particles:

$$
A=\frac{\psi^{*}-\Phi^{*}}{\left(1-\psi^{*}\right) \Phi^{*}}
$$

where $\Phi^{*}=0.64$ and $\psi^{*}=0.842$ are the random packing volume fraction for spheres, and random packing area fraction for disks, respectively, ${ }^{42}$ giving $\mathrm{A}=1.9976 \approx 2$. Equation 10 was fitted to the experimental data with adjustable parameter $\mathrm{B}$. The result shown in Figure $9 \mathrm{~b}$ was obtained with $\mathrm{B}=11.3$, which is the highest value for $\mathrm{B}$ among those reported in former studies of particulate composites. ${ }^{41,43}$ The high value of B undeniably indicates the presence of strong interactions at the particlepolymer interface, most probably arising from the covalent linkages forming between acrylic groups present at the particle surface and in the polymer upon UV-curing. 


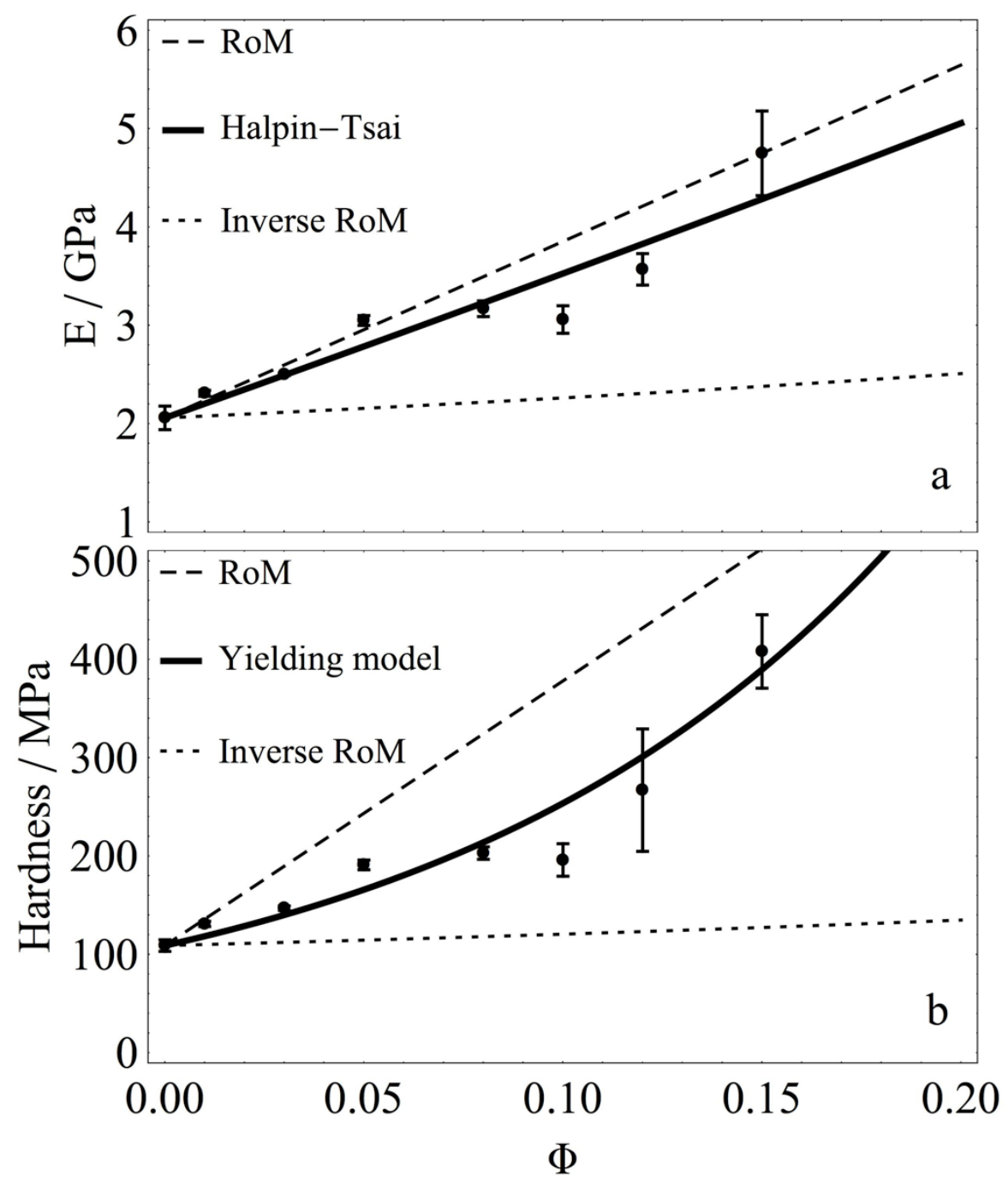

Figure 9. Young's modulus (a) and hardness (b) of nanocomposites as a function of their nanoparticle volume fraction. Dots represent experimental data whereas lines refer to the indicated models.

Analyses of the nanoindentation tests seem to indicate efficient bond formation between the nanoparticles and the acrylated matrix, further enhancing the intrinsic improvement of characteristics deriving from the inclusion of the nanofiller. Further studies to evaluate the effect of particle surface treatment on the mechanical properties of the resulting composites are under way.

\section{Conclusions}

The synthetic approach presented here provides guidance toward a smarter design of soft materials characterized by a tunable degree of local reinforcement. By filling a polymeric matrix with core-shell nanoparticles comprising a magnetic core and a silica shell with tailored surface, the magnetic responsive filler is concentrated in specific regions of the composite upon application of magnetic field gradients, ending up with non-uniform structures characterized by graded mechanical properties. It is shown how the gradient formation process is strongly dependent on both the applied magnetic force and the viscosity of the nanoparticulate suspension. In particular, it is demonstrated how the use of a simple set-up composed of two block magnets in 
repulsion configuration can increase the force applied on the magnetic filler by $24 \%$ compared to that generated by a single magnet, and how functionalization of the particle surfaces with an acrylated silane leads to a 6-fold decrease in the low shear rate complex viscosity of the nanosuspensions. Both strategies showed to be fundamental to accelerate the gradient formation process and achieve gradient morphologies otherwise unattainable. The as-synthesized materials exhibit continuous gradations in mechanical properties and show remarkable increments in elastic modulus (up to $\approx 70 \%$ ) and hardness (up to $\approx 150 \%$ ) when going from particledepleted to particle-enriched regions, even after magnetic field gradient application times as short as 1 hour.

Not only the proposed strategy merges into a single-step process the synthesis of graded structures able to maximize the reinforcing role of the filler and rationalize its utilization, but intrinsically adds functionality to the resulting nanocomposite, letting foresee interesting applications in fields such as those of magnetic sensors and actuators. In addition, owing to the multifunctionality of the core-shell filler, the applicability of the process could be extended well beyond that of bio-mimicking structures, opening up new paths to the efficient synthesis of materials with graded electrical or optical properties.

\section{Acknowledgements}

The authors acknowledge the Swiss National Science Foundation (project 200020_144396) for financial support. Sincere acknowledgements also to Manuel Bianco for his help on the construction of the permanent magnet set-up, to Danièle Laub and Colette Vallotton (CIME-EPFL) for their help on the preparation of the samples for the SEM, and to Christopher J. Plummer (LTC-EPFL) for the fruitful scientific advices.

\section{References}

[1] A.K. Ray, S.K. Das, S. Mondal, P. Ramachandrarao, J. Mater. Sci., 2004, 39, 1055.

[2] J.E. Phillips, K.L. Burns, J.M. Le Doux, R.E. Guldberg, A.J. García, PNAS, 2008, 105, 12170.

[3] A.G. Mikos, S.W. Herring, P. Ochareon, J. Elisseeff, H.H. Lu, R. Kandel, F.. Schoen, M. Toner, D. Mooney, A. Atala, M.E. Van Dyke, D. Kaplan, G. Vunjak-Novakovic, Tissue Eng., 2006, 12, 3307.

[4] A.R. Studart, Adv. Funct. Mater., 2013, 23, 4423.

[5] H. Yao, M. Dao, T. Imholt, J. Huang, K. Wheeler, A. Bonilla, S. Suresh, C. Ortiz, PNAS, 2010, 107, 987.

[6] C. Hedegaard, J. Mollus. Stud., 1997, 63, 369.

[7] H. Moshe-Drezner, D. Shilo, A. Dorogoy, E. Zolotoyabko, Adv. Funct. Mater., 2010, 20, 2723.

[8] S. Suresh, A. Mortensen, Fundamentals of Functionally Graded Materials, Maney, Leeds, UK 1998.

[9] S. Suresh, Science, 2001, 292, 2447.

[10] P.J. Hogg, Science, 2006, 314, 1100.

[11] E.D. Wachsman, K.T. Lee, Science, 2011, 334, 935. 
[12] M. Riabkina-Fishman, E. Rabkin, P. Levin, N. Frage, M.P. Dariel, A. Weisheit, R. Galun, B.L. Mordike, Mater. Sci. Eng. A, 2001, 302, 106.

[13] G. Zuccarello, D. Scribner, R. Sands, L.J. Buckley, Adv. Mater., 2002, 14, 1261.

[14] U. Schulz, M. Peters, Fr.-W. Bach, G. Tegeder, Mater. Sci. Eng. A, 2003, 362, 61.

[15] V. Birman, L.W. Byrd, Appl. Mech. Rev., 2007, 60, 195.

[16] R.K. Singh, D.R. Gilbert, S. Harkness, D.G. Lee, Science, 1996, 272, 396.

[17] J.L. Maxwell, M. Boman, R.W. Springer, A. Nobile, K. DeFriend, L. Espada, M. Sandstrom, D. Kommireddy, J. Pegna, D. Goodin, Adv. Func. Mater., 2005, $15,1077$.

[18] M. Belmonte, J. Gonzalez-Julian, P. Miranzo, M.I.Osendi, Acta Mater., 2009, 57, 2607.

[19] M. Bhattacharyya, A.M. Kumar, S. Kapuria, Mater. Sci. Eng. A, 2008, 487, 524.

[20] R. Libanori, R.M. Erb, A. Reiser, H. Le Ferrand, Hortense, M.J. Süess, R. Spolenak, A.R. Studart, Nat. Commun., 2012, 3, 1.

[21] S. Put, J. Vleugels, O. Van Der Biest, Acta Mater., 2003, 51, 6303.

[22] T. Sehayek, T. Bendikov, A. Vaskevich, I. Rubinstein, Adv. Funct. Mater., 2006, 16, 693.

[23] T. Ishikawa, H. Yamaoka, Y. Harada, T. Fujii, T. Nagasawa, Nature, 2002, 416, 64.

[24] M. Ito, T. Sakamoto, M. Suzuki, Materials Science Forum, 2009, 631-632, 161.

[25] R.C. O'Handley, Modern magnetic materials: principles and applications, Wiley, Hoboken, NJ, USA 2000.

[26] M.A.M. Gijs, F. Lacharme, U. Lehmann, Chem. Rev., 2010, 110, 1518.

[27] T. Nardi, M. Sangermano, Y. Leterrier, P. Allia, P. Tiberto, J.-A. E. Månson, Polymer, 2013, 54, 4472.

[28] W.C. Oliver, G.M. Pharr, J. Mater. Res., 1992, 7, 1564.

[29] C. Klingshirn, M. Koizumi, F. Haupert, H. Giertzsch, K. Friedrich, J. Mater. Sci. Lett., 2000, 19, 263.

[30] H. Chen, E. Ruckenstein, J. Colloid Interface Sci., 2011, 363, 573.

[31] H. Chen, E. Ruckenstein, J. Chem. Phys., 2009, 131, 244904.

[32] V. Geiser, Y. Leterrier, J.-A. E. Månson, Macromolecules, 2010, 43, 7705.

[33] G.H. McKinley, Transport Processes in Bubbles, Drops and Particles, Taylor \& Francis, New York, NY, USA 2001.

[34] A.S. Ivanov, A.F. Pshenichnikov, J. Magn. Magn. Mater., 2010, 322, 2575.

[35] L. Zhang, M. D'Acunzi, M. Kappl, G.K. Auernhammer, D. Vollmer, C.M. van Kats, A. van Blaaderen, Langmuir, 2009, 25, 2711.

[36] J.C. Halpin, J.L. Kardos, J. Appl. Phys., 1972, 43, 2235.

[37] J.C. Halpin, J.L. Kardos, Polym. Eng. Sci., 1976, 16, 344.

[38] A. Lonjon, P. Demont, E. Dantras, C. Lacabanne, J. Non-Cryst. Solids, 2012, 358, 236.

[39] R.W. Rice, P.W. McMillan, A.J. Stryjak, J. Mater. Sci., 1979, 14, 2768.

[40] M. Zou, D. Yang, Tribol. Lett., 2006, 22, 189.

[41] M.A. Gonzalez Lazo, R. Teuscher, Y. Leterrier, C. Calderone, J.-A. E. Månson, A. Hessler-Wyser, P. Couty, Y. Ziegler, D. Fischer, Sol. Energy Mater. Sol. Cells, 2012, 103, 147.

[42] K.W. Desmond, E.R. Weeks, Phys. Rev. E., 2009, 80, 051305.

[43] B. Turcsányi, B. Pukánszky, F. Tüdõs, J. Mater. Sci. Lett., 1988, 7, 160. 


\section{Supporting Information}

\section{COMPUTATION OF THE MAGNETIC FIELDS AND RELATED FORCES}

The magnetic flux densities outside the block magnets were determined by the following equations ${ }^{1}$

$$
\begin{gathered}
\bar{B}_{x}(x, y, z)=\frac{\mu_{0} \bar{M}_{s}}{4 \pi} \sum_{k=1}^{2} \sum_{m=1}^{2}(-1)^{k+m} \ln \left[\frac{\left(y-y_{1}\right)+\left[\left(x-x_{m}\right)^{2}+\left(y-y_{1}\right)^{2}+\left(z-z_{k}\right)^{2}\right]^{1 / 2}}{\left(y-y_{2}\right)+\left[\left(x-x_{m}\right)^{2}+\left(y-y_{2}\right)^{2}+\left(z-z_{k}\right)^{2}\right]^{1 / 2}}\right] \\
\bar{B}_{y}(x, y, z)=\frac{\mu_{0} \bar{M}_{s}}{4 \pi} \sum_{k=1}^{2} \sum_{m=1}^{2}(-1)^{k+m} \ln \left[\frac{\left(x-x_{1}\right)+\left[\left(x-x_{1}\right)^{2}+\left(y-y_{m}\right)^{2}+\left(z-z_{k}\right)^{2}\right]^{1 / 2}}{\left(x-x_{2}\right)+\left[\left(x-x_{2}\right)^{2}+\left(y-y_{m}\right)^{2}+\left(z-z_{k}\right)^{2}\right]^{1 / 2}}\right] \\
\bar{B}_{z}(x, y, z)=\frac{\mu_{0} \bar{M}_{s}}{4 \pi} \sum_{k=1}^{2} \sum_{m=1}^{2} \sum_{n=1}^{2}(-1)^{k+m+n} \operatorname{ArcTan}\left[\frac{\left(x-x_{n}\right)\left(y-y_{m}\right)}{\left(z-z_{k}\right)}\left[\left(x-x_{n}\right)^{2}+\left(y-y_{m}\right)^{2}+\left(z-z_{k}\right)^{2}\right]^{-1 / 2}\right]
\end{gathered}
$$

where $B_{x}, B_{y}$ and $B_{z}$ are the magnetic fields in the $x, y$ and $z$ directions, respectively, $\mu_{0}$ is the vacuum permeability, $\mathrm{Ms}$ is the saturation magnetization of the magnet and ( $\mathrm{x}_{1}$, $\left.\mathrm{x}_{2}\right),\left(\mathrm{y}_{1}, \mathrm{y}_{2}\right)$ and $\left(\mathrm{z}_{1}, \mathrm{z}_{2}\right)$ are the positions of the edges of the magnet, which is magnetized along the z-axis. The magnetic flux densities (verified by a Hall probe magnetometer), magnetic field gradients and magnetic forces were evaluated setting $\mathrm{M}_{\mathrm{s}}=10.5 \cdot 10^{5}$ $\mathrm{A} / \mathrm{m}$ and $\mathrm{x}_{1}=-0.02, \mathrm{x}_{2}=0.02, \mathrm{y}_{1}=-0.02, \mathrm{y}_{2}=0.02, \mathrm{z}_{1}=-0.02, \mathrm{z}_{2}=0$. This was done in order to ascertain which magnets arrangements give rise to the highest $\mathrm{z}$ component of $\mathrm{F}_{, \mathrm{m}}^{-}$, which is the driving force for the gradient formation along the $\mathrm{z}$-axis. For the single magnet case, the $\mathrm{z}$ component of $\mathrm{F}^{-} \mathrm{m}$ follows the behavior of the gradient of the magnetic induction along $\mathrm{z}$ and goes through a maximum positioned $2 \mathrm{~mm}$ away from the surface of the magnet (Figure S1). An increment in magnetic force can be achieved when two magnets in repulsion configuration are employed because in this case the gain in the gradient of the magnetic flux density $d B_{z}$ (Figure S1) can overcome the loss in the nanoparticle magnetic moment $m$ (due to the decrease in magnetic flux density), resulting in an higher magnetic force compared to the single magnet case. On the contrary, when two magnets in attraction configuration are employed the increment in the particle magnetic moment (due to the increment in magnetic induction intensity) can only partially compensate the loss in the modulus of the induction gradient, and the $\mathrm{z}$ component of the magnetic force will tend at best, at quite large distances $d$ between the magnets, to that generated by a single magnet. 


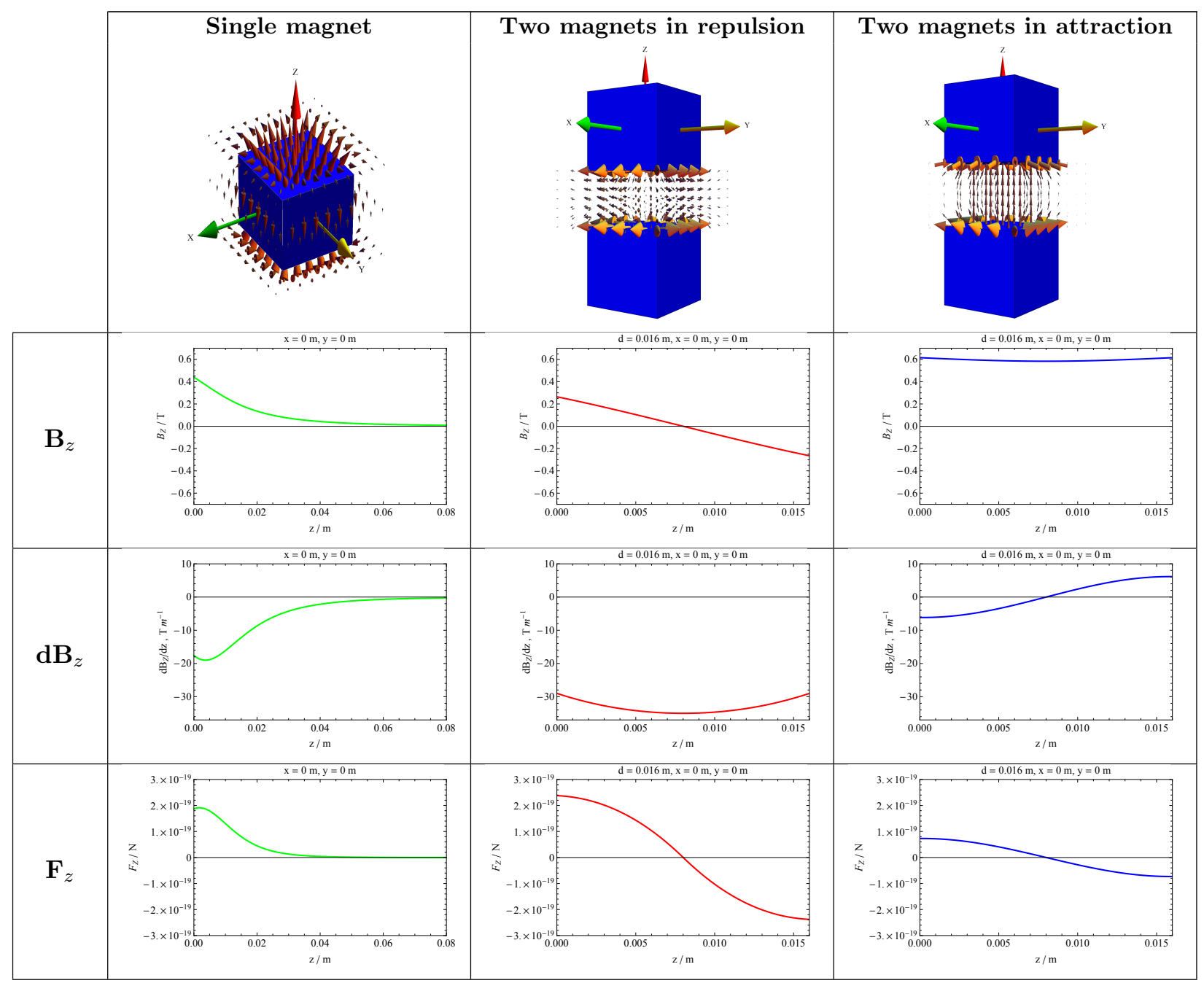

Figure S1. Table comparing the magnetic induction B, its gradient $d B$ with respect to the $z$-direction and the magnetic force $\mathrm{F}$ exerted on a single $\mathrm{Fe}_{3} \mathrm{O}_{4}$ nanoparticle for the single magnet case and the cases of two magnets in repulsion and attraction configurations. All the quantities are considered along the direction over which the gradient is wanted (z-direction).

\section{PREPARATION OF THE NANOPARTICULATE THIN FILMS}

Considering the case of the two magnets in repulsion configuration, we can see (Figure S2-a) that a much higher z-component of the magnetic force is generated towards the edges of the magnet, which spans the region from $x=-0.02 \mathrm{~m}$ to $\mathrm{x}=0.02 \mathrm{~m}$. In that same region, however, even the $\mathrm{x}$-component (and for symmetry also the y-component) of $F_{m}$ shows a noticeable increase (Figure S2-b), eventually overcoming the force acting in the z-direction upon reaching the edges of the magnet and making the formation of the gradient along the z-axis less controllable. 

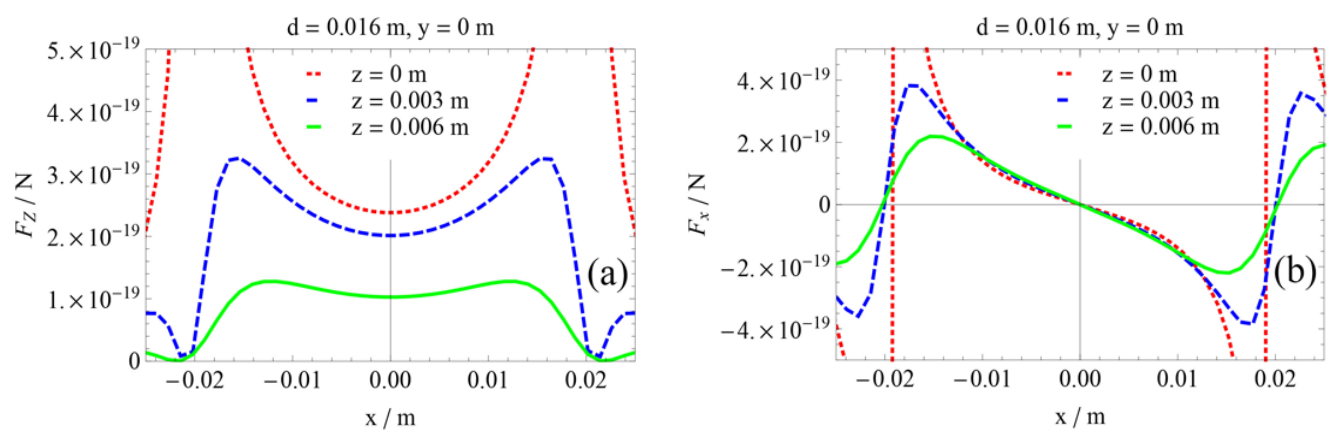

Figure S2. Plot of z- (a) and $x$ - (b) components of the magnetic force $\mathrm{F}_{m}$ acting on a $\mathrm{Fe}_{3} \mathrm{O}_{4}$ nanoparticle placed at different distances $z$ from the magnet for the case of two magnets in repulsion configuration. The two magnets are placed at a distance $d=1.6 \mathrm{~cm}$ from each other.

It was therefore important to define a central area in which the z-component of the magnetic force was predominant over the $\mathrm{x}$ - and $\mathrm{y}$ - ones. In particular, the sample was confined on a central area $(1 \mathrm{~cm} \times 1 \mathrm{~cm})$ over the bottom magnet (Figure S3) on which, point-by-point, the magnitude of the x-component of the magnetic force was always smaller than half of the magnitude of the z-component.

By doing so, the gradient formation along the $\mathrm{z}$-direction was not hindered by the action of the $\mathrm{x}$ - and $\mathrm{y}$ - components of the magnetic force.

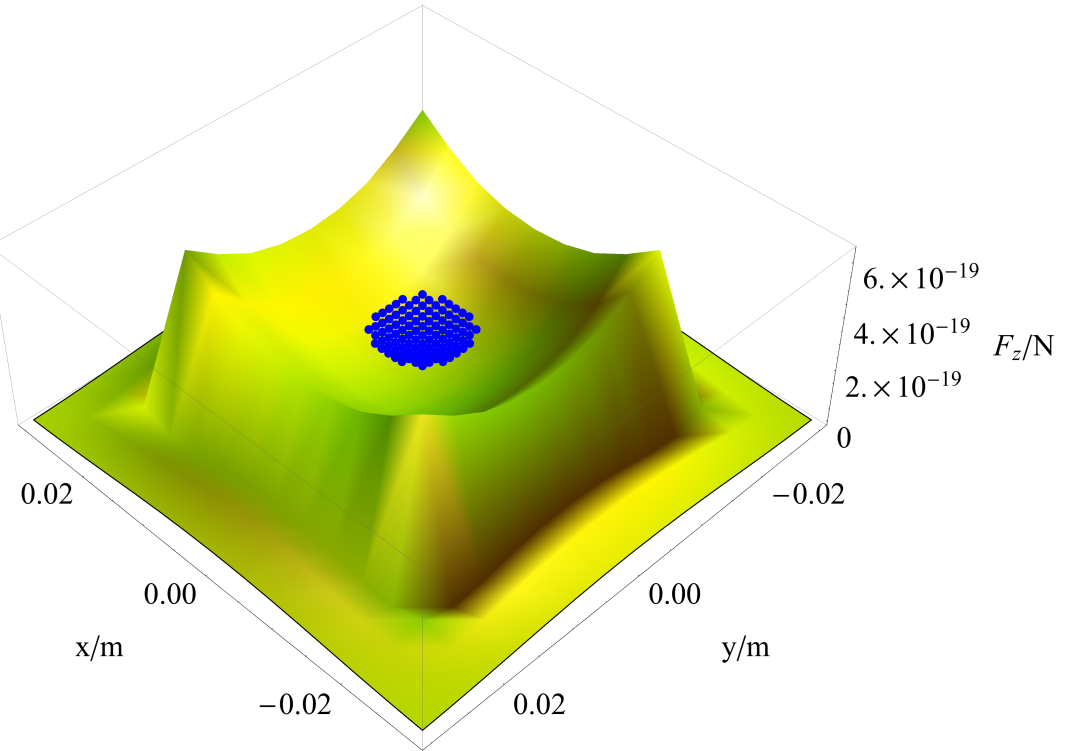

Figure S3. 3D-plot of z-component of the magnetic force $\mathrm{F}_{m}$ exerted on a $\mathrm{Fe}_{3} \mathrm{O}_{4}$ nanoparticle placed at $z=0$ between two magnets at a distance $d=1.6 \mathrm{~cm}$. The blue points define the area over which $F_{x} \leq 0.5$ $F_{z}$. 


\section{IR ANALYSIS OF Fe ${ }_{3} \mathrm{O}_{4} @$ SILICA AND Fe $\mathrm{O}_{4} @$ SILICA-MPS NANOPARTICLES}

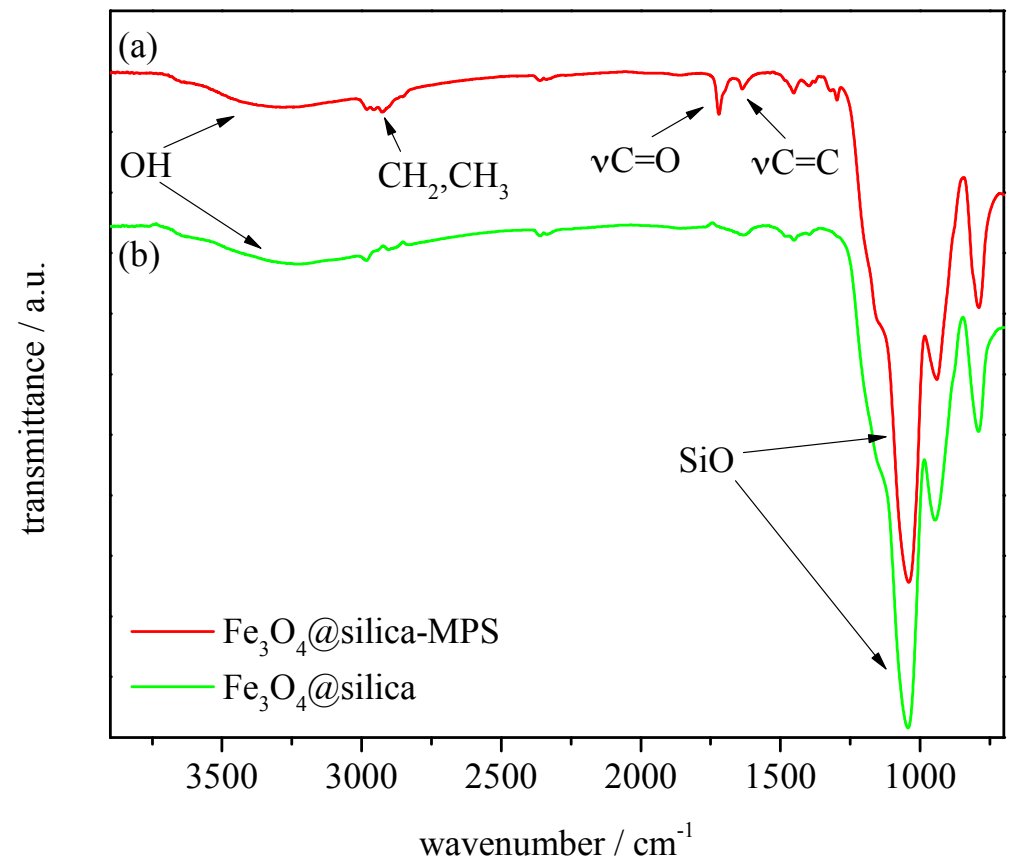

Figure S4.FT-IR spectrum of the non-functionalized (a) and MPS-functionalized (b) $\mathrm{Fe}_{3} \mathrm{O}_{4} @$ silica nanoparticles.

The FTIR spectra of the particles show absorption bands arising from asymmetric vibration of Si-O $\left(1050 \mathrm{~cm}^{-1}\right)$, asymmetric vibration of Si-OH $\left(945 \mathrm{~cm}^{-1}\right)$, and symmetric vibration of Si-O $\left(795 \mathrm{~cm}^{-1}\right){ }^{2}$ The broadband centered between $3300 \mathrm{~cm}^{-1}$ and $3500 \mathrm{~cm}^{-1}$ is assigned to the fundamental stretching vibrations of different hydroxyl groups, such as those due to adsorbed water. ${ }^{2}$ The peaks at $2945 \mathrm{~cm}^{-1}\left(\mathrm{CH}_{3}\right)$ and 2915 $\mathrm{cm}^{-1}\left(\mathrm{CH}_{2}\right)$ can be used to identify either the presence of MPS onto the silica surface and unreacted TEOS in the particles. ${ }^{3}$ As a matter of fact, these peaks are more evident for MPS-functionalized nanoparticles, supporting the first hypothesis. The presence in the spectrum of MPS-functionalized core-shell nanoparticles (a) of the two peaks at $1720 \mathrm{~cm}^{-1}$ and $1637 \mathrm{~cm}^{-1}$ assigned to $\mathrm{C}=\mathrm{O}$ and $\mathrm{C}=\mathrm{C}$ stretching, ${ }^{4}$ respectively, indicates the goodness of the proposed functionalization procedure.

[1] E.P. Furlani, Permanent magnet \& electromechanical devices: materials, analysis and applications, Academic press, San Diego, CA, USA 2001.

[2] Y. Ding, X. Chu, X. Hong, P. Zou, Y. Liu, Appl. Phys. Lett., 2012, 100, 013701.

[3] K.M. Bratlie, H. Lee, K. Komvopoulos, P. Yang, G.A. Somorjai, Nano Lett., 2007, 7, 3097.

[4] L.-V. Daimay, B.C. Norman, W.G. Fateley, J.G. Grasselli, The Handbook of Infrared and Raman Characteristic Frequencies of Organic Molecules, Academic press, London, UK 1991. 